\title{
Volksgesetzgebung: Königsweg oder Irrweg der direkten Demokratie?
}

von Fabian Wittreck

In jüngerer Zeit widmet sich die öffentliche Debatte angesichts lautstark vortragender und inzwischen milieuübergreifend verwurzelter Bürger- und Protestbewegungen verstärkt der Frage, ob das deutsche Repräsentationssystem erweiterter direktdemokratischer Elemente bedarf. Im Folgenden soll diese Problematik aus verfassungsrechtlicher Perspektive in zwei Schritten erörtert werden. Zunächst gilt es, die bestehenden Vorgaben für eine mögliche Volksgesetzgebung zu skizzieren, um anschließend die unterschiedlichen Instrumente der direkten Demokratie knapp zu bewerten. Da über die verfassungsrechtlichen Kernfragen kein ernsthafter Streit mehr bestehen kann, sollte die Einführung und Ausgestaltung derartiger Beteiligungsformen eher im Modus von „empfehlenswert“ oder ,wenig hilfreich“ diskutiert werden. Unter dieser Vorgabe werden die Kernaussagen des vorliegenden Beitrags abschließend in zehn Thesen summiert.

On the background of a growing and broadly-based culture to engage in vocal protest movements, public discourse has recently focused on the question whether the German system of representative government requires further elements of direct democracy. The following article seeks to address this problem from the perspective of constitutional law. First, the existing constitutional provisions for a possible extension of direct democratic elements will be discussed, leading, second, to an evaluation of the different available instruments. Since the debate on the core questions of constitutional law regarding direct democracy is largely settled, the introduction and the design of such forms of participation should rather be discussed in the mode of "recommendable" and "unhelpful". Based on this premise, ten concluding observations will summarise the argument presented.

\section{Direkte Demokratie im Schmollwinkel?}

Direkte Demokratie ist - längst über „Stuttgart 21“ und den Juchtenkäfer hinaus - in aller Munde und wird entweder als der Ausweg aus der gefühlten Blockade der Republik apostrophiert ${ }^{1}$ oder aber gezielt in der Hoffnung eingefordert, un-

Für Hinweise und Anregungen dankt der Verf. neben den Mitgliedern des Arbeitskreises ,Zukunft der Parteiendemokratie“ der Friedrich-Ebert-Stiftung Professor Dr. Horst Dreier und PD Dr. Otmar Jung.

1 In diesem Sinne zuletzt das Plädoyer von Gross, A.: Heilung durch direkte Demokratie, in: FAZ.net, 15.12.2010. Vgl. auch - aus der Beobachterperspektive - Köcher, R.: Der Ruf nach dem Plebiszit, FAZ Nr. 244, 20.10.2010, 5 . 
liebsame Projekte ganz im Gegenteil verhindern zu können. ${ }^{2}$ Der Eindruck des Verfassers geht dahin, dass die zweite Schwingung derzeit stärker zu sein scheint.

Das folgende verfassungsrechtliche statement zu dieser Problematik wird sich in zwei Schritte gliedern: Zuerst gilt es, die verfassungsrechtlichen Vorgaben für eine mögliche Volksgesetzgebung zu skizzieren (II.), ${ }^{3}$ bevor eine knappe Bewertung der verschiedenen in Betracht kommenden bzw. augenblicklich diskutierten Instrumente der direkten Demokratie erfolgt (III.). ${ }^{4}$

Eine Vorwegnahme und eine Vorbemerkung: ${ }^{5}$

- Zunächst gilt es die gestellte Frage zu beantworten: Einen Königsweg kann es in der „Berliner Republik“ nicht geben, und vielleicht ist das auch gut so. Der parlamentarische wie der Volksgesetzgeber haben unlängst eindrucksvoll bewiesen, dass sie das Gemeinwohlziel verfehlen und Minderheiten entweder dolos begünstigen oder dumpf bedrücken können - verringerte Mehrwertsteuersätze und verbotene Minarette mögen hier als Stichworte genügen. ${ }^{6}$ Gleiches gilt, wenn man die regelrechte Volksgesetzgebung in Gestalt von Volksbegehren und Volksentscheid mit anderen Beteiligungs-

2 So erkennbar die Tendenz des von der SPD im Landtag von Baden-Württemberg in Auftrag gegebenen Gutachtens aus der Feder von Hermes, G./Wieland, J.: Rechtliche Möglichkeiten des Landes BadenWürttemberg, die aus dem Finanzierungsvertrag ,Stuttgart 21“ folgenden Verpflichtungen durch Kündigung oder gesetzliche Aufhebung auf der Grundlage eines Volksentscheides zu beseitigen, in: Feld, L.P. u.a. (Hg.): Jahrbuch für direkte Demokratie 2010, 2011, i.E. Grundsätzlich kritisch Kirchhof, P.: Zur Verfassungsmäßigkeit einer Volksabstimmung über „Stuttgart 21“, in: ZSE 8 (2010), $411 \mathrm{ff}$. Allgemein zum Streit um das Projekt jetzt Schönenbroicher, K.: Irritationen um „Stuttgart 21“. Aktuelle Anmerkungen zu Verfahren und Bürgerbeteiligung bei raumbedeutsamen Großvorhaben, in: VBIBW 2010, $466 \mathrm{ff}$.

3 Vgl. Thesen 3-6 am Ende dieses Beitrags.

4 Vgl. Thesen 7-10.

5 Vgl. Thesen 1-2.

6 Vgl. zum ersten Punkt statt aller Neubacher, A./Wassermann, A.: Ein bisschen gaga. Die schwarz-gelbe Koalition beschließt einen Steuernachlass auf Hotelübernachtungen. Es ist der Sieg einer Lobby gegen alle ökonomische Vernunft, in: Der Spiegel 47/2009, 82. Aus der Diskussion um die Minarettinitiative seien hier genannt: Kolb, R.: Völkerrecht und Völkerpolitik. Gedanken zur Minarettinitiative, in: Schweizerische Zeitschrift für internationales und europäisches Recht 19 (2009), 467 ff.; Kiener, R.: Bedeutungswandel des Rechtsstaats und Folgen für die (direkte) Demokratie am Beispiel völkerrechtswidriger Volksinitiativen, in: Schweizerisches Zentralblatt für Staats- und Verwaltungsrecht 110 (2009), 237 ff.; Heußner, H. K.: Minarettverbot in der Schweiz: Argument gegen Volksentscheide in Deutschland? - Zur Diskussion über Volksgesetzgebung, Minderheitenschutz und Völkerrecht, in: RuP 46 (2010), 19 ff.; Nolte, J.: Kann der Souverän rechtswidrig handeln? - Eine verfassungsvergleichende Betrachtung zum Umfang der verfassungsändernden Gewalt anlässlich des schweizerischen Minarettverbots, in: DÖV 2010, 806 ff.; Strahm, D.: Feministische Nachlese zur Minarettabstimmung in der Schweiz, in: Femina Politica 19/1 (2010), 118 ff.; Antonsich, M./Jones, P. I.: Mapping the Swiss referendum on the minaret ban, in: Political Geography 29 (2010), 57 ff.; Biaggini, G.: Die schweizerische direkte Demokratie und das Völkerrecht - Gedanken aus Anlass der Volksabstimmung über die Volksinitiative „Gegen den Bau von Minaretten“, in: ZÖR 65 (2010), 325 ff.; zuletzt Tschentscher, A./ Blonski, D.: Direkte Demokratie in der Schweiz. Jahresbericht 2009/2010, in: Feld, L.P. u.a., a.a.O. 
formen der direkten Demokratie wie den verschiedenen Typen von Referenden vergleicht. ${ }^{7}$ Begreift man Demokratie als ,riskante Ordnung “, 8 so haben wir eigentlich nur die Wahl zwischen Irrwegen.

- Die Auswahl, welchem davon man folgen will - dies die Vorbemerkung muss in Deutschland endlich als genuin verfassungspolitische begriffen und diskutiert werden. ${ }^{9}$ Die deutsche Diskussion zeichnet sich nach Beobachtung des Verfassers nach wie vor dadurch aus, dass das „Ob“ und das „Wie“ direkter Demokratie gleichermaßen in der binären Codierung von ,erlaubt“ und ,verboten“" erörtert werden. Die Abgrenzung der dabei geäußerten ,verfassungsrechtlichen Bedenken“ zu politischen Präferenzen ist bezeichnenderweise latent osmotisch, nimmt mitunter auch Züge des Raunens an. ${ }^{10}$

Der Verfasser möchte demgegenüber darlegen, dass über die verfassungsrechtlichen Kernfragen der Volksgesetzgebung vernünftigerweise kein Streit mehr bestehen kann. Wir sollten über ihre Einführung und Ausgestaltung daher im Modus von „empfehlenswert“ oder „,wenig hilfreich“ diskutieren. Unter die letzte Kategorie fällt leider die augenblickliche öffentliche Debatte: So erfreulich die Aufmerksamkeit für das Thema „Direkte Demokratie“ sein mag, so offensichtlich scheint es zu sein, dass die erkennbar parteitaktischen Erwägungen wie die einseitige Suche nach „Verhinderungsoptionen“ einer verfassungspolitischen Prüfung sine ira et studio nicht förderlich sind.

\section{Der Spielraum des verfassungsändernden Gesetzgebers}

Zunächst aber zu den verbleibenden verfassungsrechtlichen Vorgaben für eine Volksgesetzgebung. ${ }^{11}$

7 Dazu jetzt kontrovers Decker, F.: Volksgesetzgebung oder Volksveto? Überlegungen zur institutionellen Ausgestaltung der Direktdemokratie in der Bundesrepublik, sowie Patzelt, W. J.: Welche plebiszitären Instrumente könnten wir brauchen? Einige systematische Überlegungen, beide in: Feld, L.P., a.a.O. Ein instruktiver aktueller Überblick zum Instrumentarium bei Neumann, P.: Regelungsbestand der Sachunmittelbaren Demokratie in Deutschland 2009, in: ders./Renger, D. (Hg.), Sachunmittelbare Demokratie im interdisziplinären und internationalen Kontext 2008/2009, 2010, 13 (15 ff.).

8 Dreier, H.: Der freiheitliche Verfassungsstaat als riskante Ordnung, in: Rechtswissenschaft 1 (2010), $11 \mathrm{ff}$.

9 Begrüßenswert daher die Perspektive von Christmann, A.: Von links geliebt - von rechts gefürchtet. Wer profitiert wirklich von direkter Demokratie?, in: Neumann, P./Renger, D., a.a.O., $256 \mathrm{ff}$.

10 Zwischen verfassungspolitischer und verfassungsrechtlicher Argumentation changiert namentlich die pointiert kritische Darstellung von Krause, P.: Verfassungsrechtliche Möglichkeiten unmittelbarer Demokratie, in: Isensee, J./Kirchhof, P. (Hg.): Handbuch des Staatsrechts der Bundesrepublik Deutschland, Bd. III, 3. Aufl. 2005, § 35 Rn. 17 ff. und passim.

11 Eingehend zum folgenden Dreier, H./Wittreck, F.: Repräsentative und direkte Demokratie im Grundgesetz, in: Feld, L.P. u.a. (Hg.): Jahrbuch für direkte Demokratie 2009, 2010, 11 ff. 
Praktisch unstrittig ist zunächst, dass die Einführung direktdemokratischer Instrumente auf Bundesebene - seien es Volksentscheid oder Referendum - nicht im Sinne von Art. 79 Abs. 3 GG die „Grundsätze“ des Demokratieprinzips ,,berühren“ würde. ${ }^{12}$ Soweit ersichtlich, haben die Stimmen, die von einer wie auch immer gearteten Präponderanz des Repräsentativsystems ausgehen, ${ }^{13}$ hier eine Frontbegradigung vorgenommen und sich darauf verlegt, nur noch Vorgaben für die Ausgestaltung zu fordern. ${ }^{14}$ Soweit vereinzelt die Auffassung vertreten worden ist, eine Volksgesetzgebung auf Bundesebene sei nicht mit der Garantie der Mitwirkung der Länder zu vereinbaren, ${ }^{15}$ dürfte der Hinweis auf die einwandfrei funktionierende Schweizer Praxis genügen. ${ }^{16}$

In umgekehrter Perspektive steht weitgehend außer Streit, dass die Einführung direktdemokratischer Elemente auf Bundesebene - geht man über eine unverbindliche bloße Volksbefragung hinaus - nicht durch einfaches Bundesgesetz erfolgen kann. ${ }^{17}$ Zwar sieht Art. 20 Abs. 2 S. 2 GG „Abstimmungen“ dem Grunde nach vor, aber die Vorschriften über die Bundesgesetzgebung namentlich in Art. 76 Abs. 1 und Art. 77 Abs. 1 GG sind in der Benennung der Akteure hinreichend präzise, um als abschließend gelten zu können. ${ }^{18}$

Ungleich heterogener wird das Meinungsbild, wenn man nach Vorgaben fragt, die mit Überverfassungsrang den verfassungsändernden Gesetzgeber bei der Detailarbeit anleiten. Geht man von den Prämissen aus, dass erstens Art. 20

12 Wie hier statt aller (m.w.N.) Dreier, H., in: ders. (Hg.): Grundgesetz-Kommentar, Bd. II, 2. Aufl. 2006, Art. 79, III, Rn. 40.

13 Prominent Böckenförde, E.-W.: Mittelbare/repräsentative Demokratie als eigentliche Form der Demokratie, in: Festschrift Eichenberger, 1982, 301 ff.; in diesem Sinne zuletzt Isensee, J.: Demokratie ohne Volksabstimmung: das Grundgesetz, in: Hillgruber, C./Waldhoff, C. (Hg.): 60 Jahre Bonner Grundgesetz - eine geglückte Verfassung?, 2010, $117 \mathrm{ff}$.

14 In diesem Sinne statt aller Isensee, J., a.a.O., 131: ,wirksame Vorkehrungen [...], so durch angemessene Quoren und Ausschluss missbrauchsanfälliger Regelungsgegenstände wie des Staatshaushalts und der Abgaben- und Leistungsgesetze."

15 So namentlich Engelken, K.: Volksgesetzgebung auf Bundesebene und die unantastbare Ländermitwirkung nach Art. 79 Abs. 3 GG, in: DÖV 2006, 550 (552 u. passim), der darauf abstellt, die grundsätzliche Mitwirkung der Länder an der Gesetzgebung i.S.v. Art. 79 Abs. 3 GG werde bei Bundes-Volksentscheiden nicht gewahrt.

16 Näher Estel, D.: Bundesstaatsprinzip und direkte Demokratie im Grundgesetz, 2006, $102 \mathrm{ff}$.

17 Dreier, H., in: ders., GGK II (Fn. 12), Art. 20 (Demokratie), Rn. 110; so auch Isensee, Demokratie ohne Volksabstimmung (Fn. 13), 130. Kritisch Jarass, H. D./Pieroth, B.: GG, 11. Aufl. 2010, Art. 20, Rn. 7.

18 Der Einwand, Art. 76 Abs. 1 und 77 Abs. 1 GG regelten lediglich das Verfahren der parlamentarischen Gesetzgebung und enthielten mithin keinerlei Aussage zur Volksgesetzgebung, ist zwar nicht leichter Hand von derselben zu weisen. Gleichwohl belegt ein Blick in die bundesdeutschen Landesverfassungen, dass diese in den entsprechenden Vorschriften stets Volks- und Parlamentsgesetzgeber explizit nebeneinander nennen (statt aller Art. 71, 72 Abs. 1 BayVerf.); für das Grundgesetz eine davon völlig losgelöste Kompositionstechnik zu postulieren, dürfte nicht sachgerecht sein. 
Abs. 1 u. 2 GG gleichrangig die Ausübung der Staatsgewalt durch Wahlen und Abstimmungen in die Hände des Volkes legt und zweitens Art. 79 Abs. 3 GG „Grundsätze“ schützt, nicht aber deren konkrete Ausgestaltung im gesamten Grundgesetz, dann ist jede Argumentation mit einem wie auch immer gearteten Vorrang des Repräsentativsystems nur um den Preis einer Metaphysik des status quo zu haben. Und jeder Hinweis auf die „Funktionsfähigkeit“ ist bivalent, muss er doch nach richtiger Ansicht zugunsten beider Spielarten der Demokratie streiten: ${ }^{19}$ In der Tat darf direkte Demokratie nicht so ausgestaltet sein, dass das Parlament seinen Aufgaben nicht mehr nachkommen kann (weit reichende Bindungsklauseln nach kalifornischem Muster sind hier ein warnendes Beispiel ${ }^{20}$ ). Nicht minder darf freilich die repräsentative Demokratie keinesfalls so ein- und zugerichtet sein, dass die Abhaltung von Volksentscheiden oder auch Referenden etwa so wahrscheinlich wird wie die endgültige Sanierung der Staatsfinanzen (das nicht minder warnende Beispiel ist hier das völlig illusorische 20-prozentige Unterstützungsquorum für ein Volksbegehren im Saarland ${ }^{21}$ ).

Legt man diese Maßgaben zugrunde, so ist der verfassungsändernde Gesetzgeber bei der Ausgestaltung etwaiger direktdemokratischer Ebenen auf Bundesebene denkbar frei. ${ }^{22}$ Als „Grundsatz“ des Demokratieprinzips im Sinne von Art. 79 Abs. 3 GG dürfte sich lediglich das Erfordernis einer Qualifikationshürde für die Unterstützung des Volksbegehrens plausibel belegen lassen. Dem Verfasser fehlt leider die Deutungsfreude einiger Kollegen der Staatsrechtslehrerzunft, die aus Ewigkeitsklauseln konkrete Zahlenangaben destillieren, ${ }^{23}$ so dass er mit einer solchen nicht aufwarten kann. Die aus dem Parlamentsrecht geläufige Fünfprozenthürde wäre jedenfalls allemal ausreichend, um ein Begehren als hinreichend gewichtig auszuweisen, die verbindliche Befragung des Souveräns einzuleiten. ${ }^{24}$

Hingegen belegen der intraföderale wie der internationale Rechtsvergleich, dass weder Quoren beim Volksentscheid noch Kataloge von Ausschlusstatbeständen

19 Nochmals Dreier, H./Wittreck, F., a.a.O., $18 \mathrm{f}$.

20 Nach kalifornischem Recht kann das Parlament vom Volk beschlossene Gesetze nur ändern, wenn das Volk dem Änderungsgesetz wiederum zustimmt; näher Heußner, H.K.: Direkte Demokratie in den USGliedstaaten im Jahr 2008, in: Feld, L.P. u.a., Jahrbuch 2009, 165 (171).

21 s. Art. 99 Abs. 2 S. 3 SaarlVerf.

22 Das Folgende im Anschluss an Wittreck, F.: Direkte Demokratie und Verfassungsgerichtsbarkeit, in: JöR 53 (2005), 111 (182 ff.).

23 Die Annahme, ein Unterstützungsquorum von nicht weniger 10\% für Volksbegehren lasse sich mit Überverfassungsrang aus Art. 83 Abs. 3 ThürVerf. (dem Thüringer Pendant zu Art. 79 Abs. 3 GG) ableiten, findet sich bei Gröschner, R.: Unterstützungsquoren für Volksbegehren: eine Frage des Legitimationsniveaus plebiszitärer Gesetzesinitiativen, in: ThürVB1. 2001, 93 (97 ff.).

24 Wie hier Patzelt, W.J., a.a.O., C.I. 
notwendig sind, um einen Staat weiterhin den legitimen Anspruch erheben zu lassen, er organisiere die Herrschaft des Volkes für das Volk und durch das Volk. ${ }^{25}$ Namentlich Quoren dienen nicht dem Schutz des Mehrheitsprinzips, sondern sind Ausnahmen der demokratischen Grundregel „Mehrheit entscheidet“, die ihrerseits vor dem Demokratieprinzip rechtfertigungsbedürftig sind. Dass dieser Entlastungsbeweis namentlich bei Beteiligungsquoren ab einer bestimmten Höhe notwendig scheitern muss, weil sie den Erfolgswert der abgegebenen Stimmen verzerren und obendrein zu Zufallsergebnissen führen, hat unlängst in Anlehnung an die Entscheidung des Bundesverfassungsgerichts zum negativen Stimmgewicht Otmar Jung eindrucksvoll dargelegt. ${ }^{26}$

In wiederum umgekehrter Perspektive sind die in Hamburg zunächst intensiv diskutierten und dann auch in die Verfassung implantierten Bindungsfristen für Volksentscheide, die diese zumindest für einen bestimmten Zeitraum praktisch parlamentsfest machen wollen, ${ }^{27}$ angesichts der jüngeren hanseatischen Erfahrungen zwar subjektiv nachvollziehbar, ${ }^{28}$ aber weder vom Demokratieprinzip geboten noch empfehlenswert. ${ }^{29}$ Ganz im Gegenteil können vergleichbare Regelungen namentlich in Kumulation mit dem Erfordernis einer qualifizierten Mehrheit zur Aufhebung volksbeschlossener Gesetze ${ }^{30}$ tatsächlich den Vorbehalt der Funktionsfähigkeit aktivieren, der zugunsten von Volks- und Parlamentsgesetzgeber gleichermaßen gelten muss.

Überlegenswert (wenn auch nicht zwingend) wäre schließlich die skrupulöse Prüfung, ob die gegenwärtig auf Landesebene begegnende verfassungsprozessuale Ungleichbehandlung volks- und parlamentsbeschlossener Gesetze sinnvoll oder gar geboten ist. ${ }^{31}$ Denn die durchweg eingeräumte Möglichkeit der Vorabprüfung von volksinitiierten Gesetzen (der hiesige Verfassungsgerichtshof hat sie

25 Einige Hinweise hierzu bei Wittreck, F., Direkte Demokratie, $176 \mathrm{ff}$.

26 Jung, O.: Zur Problematik des Beteiligungsquorums, in: Feld, L.P. u.a, Jahrbuch 2009, 40 (41 ff.) im Anschluss an BVerfGE 121, 266.

27 Art. 50 Abs. 2 HambVerf. sieht vor, dass von der Bürgerschaft beschlossene Änderungsgesetze zu volksbeschlossenen Gesetzen erst nach drei Monaten in Kraft treten; in dieser Zeit können zweieinhalb Prozent der Stimmberechtigten einen Volksentscheid über das Änderungsgesetz herbeiführen. Näher $v$. Arnauld, A.: „Refolution“ an der Elbe: Hamburgs neue direkte Demokratie - Die Verfassungsänderungen der Jahre 2008 und 2009 im Kontext, in: Feld, L.P. u.a., Jahrbuch 2009, 90 (115 ff.).

28 Die Auseinandersetzungen schildert v. Arnauld, a.a.O., $96 \mathrm{ff}$.

29 Vgl. Jung, O.: Volksgesetze und parlamentarische Konterlegislatur, in: Schrenk, K. H./Soldner, M. (Hg.): Analyse demokratischer Regierungssysteme. Festschrift Ismayr, 2010, 427 ff.

30 Vgl. nochmals das mahnende Beispiel Kalifornien (Fn. 20).

31 Näher zum Verhältnis von direkter Demokratie und Verfassungsgerichtsbarkeit Wittreck, F.: Direkte Demokratie, a.a.O., 116 ff.; ders., Direkte Demokratie vor Gericht, in: Heußner, H.K./Jung, O. (Hg.): Mehr direkte Demokratie wagen, 2. Aufl. 2009, 397 ff. 
letztes Jahr erfreulich restriktiv gehandhabt ${ }^{32}$ ) stellt diese nicht nur in durchaus fragwürdiger Weise unter den Generalverdacht, weniger gemeinwohlorientiert zu sein als parlamentarische Initiativen, sondern wirft auch eine strukturelle Machtfrage auf: Volksbegehren können auf diese Weise zu einem Zeitpunkt gestoppt werden, zu dem sie noch nicht das Unterstützungs- und eben auch Drohpotential aufgebaut haben, das den politischen „Preis“ ihrer Verwerfung in die Höhe treibt (aber natürlich achten Verfassungsrichter nicht auf solche Preisschilder). ${ }^{33}$

In umgekehrter Perspektive belegen zahlreiche Verfahren, dass für Streitigkeiten im Vorfeld eines Volksbegehrens oder -entscheids nicht durchgehend der (verfassungsgerichtliche) Rechtsschutz zur Verfügung steht, der der Organstellung des Volkes angemessen wäre. ${ }^{34}$

\section{Instrumente der Bürgerbeteiligung im Vergleich}

Kommen wir abschließend zu den konkreten Regelungsoptionen direktdemokratischer Beteiligung. Hier steht zunächst die Weichenstellung „Volksbegehren oder bloßes Referendum“ an. Der Verfassung ist dazu keinerlei Orientierung zu entnehmen; beide Spielarten würden - vorbehaltlich gravierender Konstruktionsfehler in der Ausgestaltung im Detail - vor Art. 79 Abs. 3 GG Bestand haben. In wohlgemerkt verfassungspolitischer Perspektive spricht gerade die eingangs geschilderte obwaltende Neigung, direkte Demokratie primär als Blockadeinstrument zu begreifen, dringend gegen eine Beschränkung der Bürger auf Referenden. Zwar trifft zumindest für die Länder der Bundesrepublik zu, dass Volksbegehren bislang überwiegend einer Gegen-Bewegung entspringen, die sich an konkreten Projekten der Repräsentanten reibt. ${ }^{35}$ Aber selbst in diesen Fällen können sich die Initiatoren eben nicht auf die Werbung für das schlichte „Nein“

32 VerfGH Berlin LKV 2009, 514 (mit Anm. von Hellriegel, M.: ebda., 516 f.).

33 Näher Wittreck, F.: Direkte Demokratie vor Gericht, a.a.O., 397 f. Die Sorge, die Gerichte bis hin zum Bundesverfassungsgericht würden vor der Aufhebung selbst evident verfassungswidriger Gesetze zurückschrecken, wenn sie sie erst nach dem Volksentscheid prüfen könnten, dürfte nicht berechtigt sein: US-amerikanische Gerichte finden sich in genau dieser Position und haben in den vergangenen Jahrzehnten bis zu einem Drittel der volksbeschlossenen Gesetze kassiert, wenn sie etwa Rechte von Minderheiten verletzten: Eingehende Darstellung von Miller, K.P.: Direct Democracy and the Courts, Cambridge u.a. 2009, 75 ff., 108 ff. und passim (vgl. insb. seine Einschätzung zum „Pre-Election Review“ (98 ff.) als ,one of the deadliest weapons“ gegen Volksinititativen (Zitat 98).

34 Instruktiver Überblick bei Mann, T.: Rechtsschutz bei Bürger- und Volksbegehren in Deutschland, in: Neumann, P./Renger, D., Sachunmittelbare Demokratie, 79 (89 ff.). - Nachahmenswert sind insofern Generalklauseln nach Art von Art. 50 Abs. 6 HambVerf. und Art. 75 Nr. 2 Sachs.-Anh. Verf. (beide Bestimmungen stellen klar, dass das Verfassungsgericht für alle Streitigkeiten aus Anlass der Durchführung von Volksinitiativen, -begehren und -entscheiden zuständig ist).

35 Eingehend bei Decker, F., a.a.O. 
beschränken, sondern müssen ihren eigenen Politikentwurf formulieren. Volksbegehren und Volksentscheid wohnt auf diese Weise wenigstens ein heilsamer Zwang zur inhaltlichen Aktivierung inne, der dem Referendum in dieser Form fehlt. Wichtiger noch ist die nach allen Ergebnissen des Rechtsvergleichs sehr wohl bestehende Chance, dass Initiativen mit eigenständigen Politikentwürfen aufwarten und Probleme thematisieren, die in der etablierten Politik schlicht vernachlässigt werden; ${ }^{36}$ schon der Bayerische Volksentscheid zum Nichtraucherschutz wäre grob verzeichnet, wollte man ihn umstandslos unter ,,Verhinderung“ buchen. ${ }^{37}$ Eine Beschränkung auf Referenden könnte vor diesem Hintergrund dem Rückzug der direkten Demokratie in den Schmollwinkel der Blockadepolitik Vorschub leisten.

Bleiben wir bei Referenden, so nehmen die fakultativen Volksentscheide eine Sonderrolle ein, die in einzelnen Bundesländern noch in Form eines Stichentscheids zwischen Landesregierung und Landesparlament begegnen. Ihre schwache Spielart (sie zeichnet sich dadurch aus, dass die Regierung eine im Parlament gescheiterte Vorlage zum Volksentscheid bringen kann, dessen Folgen sich jedoch im Erfolgsfall auf das Zustandekommen des Gesetzes beschränken) ist in Baden-Württemberg gerade Grundlage für Gedankenspiele zu „Stuttgart 21“.38 Die starke Variante (beheimatet etwa in Nordrhein-Westfalen) macht den Volksentscheid über ein vom Landtag abgelehntes Gesetz zugleich zum Plebiszit über den Fortbestand von Landtag oder Regierung; ${ }^{39}$ in funktionaler Perspektive tritt das Volk hier weniger als Gesetzgeber denn als Austrägalgericht in Erschei-

36 Engagiert vorgetragen von Patzelt, W.J., a.a.O.

37 Erste Besprechungen: Groß, R.: Der Volksentscheid zum Nichtraucherschutz in Bayern, in: Kommunalpraxis Bayern 2010, 170 ff.; Scheidler, A.: Nichtraucherschutz in bayerischen Gaststätten nach dem Volksentscheid vom 4.7.2010, in: BayVB1. 2010, 645 ff.; Weixner, B. M.: Nichtraucherschutz in Bayern - der Weg eines erfolgreichen Volksbegehrens und Volksentscheids im Freistaat, in: Feld, L.P. u.a., Jahrbuch 2010; Schoen, H./Glantz, A./Teusch, R.: Abstimmungskampf, Informationsvermittlung und Stimmentscheidung beim Volksentscheid über den Nichtraucherschutz in Bayern, ebd.

38 Konkret sieht Art. 60 Abs. 3 Bad.-Württ. Verf. vor, dass ,die Regierung eine von ihr eingebrachte, aber vom Landtag abgelehnte Gesetzesvorlage zur Volksabstimmung bringen“ kann, ,[w] enn ein Drittel der Mitglieder des Landtags es beantragt“ (vgl. dazu allgemein Braun, K.: Kommentar zur Verfassung des Landes Baden-Württemberg, 1984, Art. 60 Rn. 16 f.). Daran schloss sich die Überlegung an, die Landesregierung könne über eine gezielt herbeigeführte Abstimmungsniederlage und einen anschließenden Antrag der Opposition den Weg zur Volksabstimmung über das Projekt öffnen.

39 Art. 68 Abs. 3 Nordrh.-Westf. Verf. räumt der Landesregierung das Recht ein, von ihr eingebrachte, vom Landtag jedoch abgelehnte Gesetze ,zum Volksentscheid zu stellen. Wird das Gesetz durch den Volksentscheid angenommen, so kann die Landesregierung den Landtag auflösen; wird es durch den Volksentscheid abgelehnt, so muss die Landesregierung zurücktreten." Vgl. dazu die Darstellungen von Mann, T., in: Löwer, W./Tettinger, P.J. (Hg.), Kommentar zur Verfassung des Landes NordrheinWestfalen, 2002, Art. 68, Rn. 46 ff. sowie zuletzt Günther, C., in: Heusch, A./Schönenbroicher, K. (Hg.), Die Landesverfassung Nordrhein-Westfalen, 2010, Art. 68, Rn. 19 ff. 
nung. ${ }^{40}$ Der Befund, dass beide Arten von Stichentscheiden bislang nicht eingeleitet worden sind, unterstreicht nur, dass wir es hier mit direktdemokratischen Artefakten des Konstitutionalismus zu tun haben, die schon in der Zwischenkriegszeit seltsam disloziert wirkten ${ }^{41}$ und im entwickelten parlamentarischen Regierungssystem mit kompetenzstarker Verfassungsgerichtsbarkeit ebenso Fremdkörper sind wie die Ministeranklage. ${ }^{42}$

Schließlich gilt es, bei der Ausgestaltung einer etwaigen Volksgesetzgebung die Erfahrungen aus der Schweiz und den USA - namentlich Kalifornien - zu beherzigen. Sie legen nahe, dass sowohl die Beschränkung der Stimmbürger auf eine Verfassungsinitiative (sie begegnet in der Schweiz auf Bundesebene, vgl. insb. Art. $139 \mathrm{BV}^{43}$ ) als auch deren ungenügende Differenzierung vom einfachen Gesetzesbegehren in puncto Einleitungsquorum (in Kalifornien genügen für die Gesetzesinitiative fünf Prozent der Stimmen der letzten Gouverneurswahl, für Verfassungsinitiativen acht $^{44}$ ) die Bürger zwingen oder doch ermuntern, politischen Anliegen sogleich Verfassungsrang zu verschaffen. Die Folgen reichen von Sünden wider den Geist der Verfassungsästhetik nach Art des notorischen Absinthverbots ${ }^{45}$ (solche sind freilich kein Privileg des Volksgesetzgebers, wie Art. 125c Abs. 2 S. 2 GG mit seinem Verweis auf $\S 6$ Abs. 1 des Gemeindeverkehrsfinanzierungsgesetzes in stiller Einfalt dokumentiert) bis hin zu handfesten Spannungen im Verfassungsgefüge infolge der punktuellen Einfügung von Regelungen, die ihrer ganzen Fallhöhe nach nicht in die Verfassung gehören, Wertungswidersprüche provozieren und schließlich Einzelpositionen festschreiben, die der fortlaufenden Aktualisierung bedürfen oder bedurft hätten. Konsequenz muss sein, dass das Verfassungsbegehren - gegen das verfassungsrechtlich wie

40 Prägnant Günther, C., a.a.O., Rn. 19.

41 Nachweise zum Stichentscheid in den Weimarer Landesverfassungen bei Wittreck, F. (Hg.): Weimarer Landesverfassungen, 2004, $49 \mathrm{f}$.

42 Einen Rest von Relevanz entfalten solche Vorschriften allerdings dann, wenn - wie derzeit in Nordrhein-Westfalen (näher Busch, J.-D.: Geschäftsregierung und Regierungsbildung im Lande NordrheinWestfalen nach der Landtagswahl am 9. Mai 2010, in: NWVB1. 2010, 373 ff.) - eine Minderheitsregierung amtiert, für die das Scheitern von Gesetzesvorlagen zum realistischen Szenario wird (so auch Braun, K., a.a.O., Rn. 16). Gleichwohl gilt auch hier, dass die ,fatale[n] Folgen“ (Günther, C., a.a.O., Rn. 19) des (verlorenen) Volksentscheids entweder von dem Rekurs auf das Instrument abhalten oder Ausweichstrategien nahelegen werden, die ein ähnliches Ergebnis (Neuwahlen) mit weniger Risiken herbeiführen.

43 Kritische Darstellung mit eingehenden Nachweisen: Tschentscher, A.: Direkte Demokratie in der Schweiz - Länderbericht 2008/2009, in: Feld, L.P. u.a., Jahrbuch 2009, a.a.O., 205 (222); vgl. aus der neueren Literatur ferner Christmann, A.: Sachunmittelbare Demokratie in der Schweiz. Überblick und aktuelle Erwägungen, in: Neumann, P./Renger, D., a.a.O., 57 (60 ff.).

44 Näher Miller, K.P., a.a.O., 3 ff., 51 ff. sowie Heußner, H.K.: Direkte Demokratie, a.a.O., 171 u. passim. 451908 als Art. 32b BV eingeführt. 
verfassungspolitisch an sich nichts einzuwenden ist - hinreichend deutlich vom Gesetzesbegehren abgesetzt werden muss, was mindestens die Verdopplung des erforderlichen Unterstützungsquorums nahe legt. ${ }^{46}$

Ernsthaft nahe treten sollte man hingegen der Prüfung einer anderen Form der direktdemokratischen Mitwirkung an der Verfassungsgesetzgebung, nämlich dem obligatorischen Verfassungsänderungsreferendum nach dem Muster der ältesten Landesverfassungen Bayerns und Hessens. ${ }^{47}$ Denn angesichts von inzwischen 58 Grundgesetzänderungen durchaus unterschiedlicher Art und Güte ${ }^{48}$ wirkt die Änderungsfrequenz in Bayern (elf) und Hessen (sieben) anheimelnd entschleunigt. ${ }^{49}$ Beide Bundesländer erwecken dabei nicht den Eindruck, sie seien gerade wegen ihres Verfassungsänderungsregimes reformunfähig. Zugleich belegen die dortigen Erfahrungen, dass im Konsens getroffene und von den Bürgern auch als hinreichend gewichtig wahrgenommene Eingriffe in die Verfassungsurkunde diese Hürde nehmen können. Mit anderen Worten: Der Verfasser ist mithin überzeugt, dass die Schuldenbremse im Verfassungsreferendum nicht blockiert hätte bzw. worden wäre. Bei Art. 91d GG, der endlich dem benchmarking verfassungsrechtliche Weihen verschafft hat, ist sich der Verfasser weniger sicher - jedoch spricht dies nicht gegen das obligatorische Verfassungsreferendum, sondern gegen Art. $91 \mathrm{~d}$ GG. ${ }^{50}$

\section{Ergebnisse in Thesen:}

(1) Demokratie wie direkter Demokratie sind Königswege gleichermaßen fremd. Volksgesetzgebung ist weder der rein parlamentarischen Legislatur

46 Insofern ist - bei aller notwendigen Kritik im Detail - die Entscheidung des Bayerischen Verfassungsgerichtshofs zumindest im Ansatz nachvollziehbar, in Abkehr von seiner älteren Rechtsprechung zu Art. 2 Abs. 2 S. 2 BayVerf. (,Mehrheit entscheidet“) im Wege der offen richterrechtlichen Setzung ein erhöhtes Quorum für Volksentscheide zu fordern, die auf Verfassungsänderungen zielen (BayVerfGHE 52, 104 [135]; 53, 42 [65 f.]). Zum Kontext der Entscheidungen wie ihren gravierenden methodischen Schwächen im übrigen (m.w.N.) Wittreck, F., Direkte Demokratie, a.a.O., 139 f., 141 ff., 145 ff.

47 Wie hier Schulze-Fielitz, H.: Schattenseiten des Grundgesetzes, in: Dreier, H. (Hg.), Macht und Ohnmacht des Grundgesetzes, 2009, 9 (17 f.). Einschlägig sind Art. 75 Abs. 2 S. 2 BayVerf. sowie Art. 123 Abs. 2 Hess Verf.

48 Zusammenfassend m.N. zuletzt Wittreck, F.: Die Entwicklung des Grundgesetzes seit 1949, in: Ad Legendum 2011, 1 (2 f. u. passim).

49 Nachweise bei Lindner, J. F., in: ders./Möstl, M./Wolff, H. A.: Verfassung des Freistaates Bayern, Vorb. A Rn. 22 bzw. Will, M.: Die Entstehung der Verfassung des Landes Hessen von 1946, 2009, $554 \mathrm{f}$.

50 Lapidar Heun, W., in: Dreier, H. (Hg.), Grundgesetz-Kommentar, 2. Aufl., Supplementum 2010, Art, 91d, Rn. 4: „.. ist die Aufnahme der Vorschrift in das Grundgesetz nicht gerechtfertigt.“ 
noch referendumsbasierten Modellen der Bürgerbeteiligung a priori überoder unterlegen.

(2) Die Einrichtung einer Volksgesetzgebung ist eine genuin verfassungspolitische Frage, die nicht länger als verfassungsrechtliche ausgeflaggt werden sollte. Zugleich leidet die notwendige verfassungspolitische Debatte, wenn sie taktisch und ohne Distanz zu konkreten Streitfällen geführt wird.

(3) Der Einführung von Volksentscheiden wie Referenden auf Bundesebene steht Art. 79 Abs. 3 GG nicht entgegen. Zugleich wäre namentlich im Hinblick auf Art. 76 und 77 GG eine solche Verfassungsänderung erforderlich.

(4) Bei der konkreten Ausgestaltung einer solchen Volksgesetzgebung ist der verfassungsändernde Gesetzgeber denkbar frei. Von Verfassung wegen geboten ist lediglich ein Unterstützungsquorum beim Volksbegehren, während weder Ausschlusstatbestände noch Quoren beim Volksentscheid zwingend sind. Ganz im Gegenteil sind namentlich Beteiligungsquoren verfassungsrechtlich bedenklich, da sie den Erfolgswert der Stimmen erheblich verzerren können.

(5) Umgekehrt sind „Sperrfristen“ zum Schutz von volksbeschlossenen Gesetzen weder von der Verfassung gefordert noch empfehlenswert.

(6) Das legitimatorische Gleichauf von Volks- und Parlamentsgesetzgebung legt nahe, beide nur einer nachgelagerten verfassungsgerichtlichen Inhaltskontrolle zu unterwerfen. Notwendig ist hingegen effektiver Rechtsschutz im Vorfeld.

(7) Die Beschränkung der direktdemokratischen Instrumente auf Referenden birgt die Gefahr, dass unmittelbare Demokratie einseitig als Blockadeoption wahrgenommen wird oder gar fungiert. Vorzugswürdig ist die Möglichkeit der aktiven bürgerlichen Gestaltung von der Initiative bis hin zum volksbeschlossenen Gesetz.

(8) Die überkommenen fakultativen Referenden in Form von „Stichentscheiden“ zwischen Regierung und Parlament sind Fremdkörper im parlamentarischen Regierungssystem und ebenso entbehrlich wie die Figur der Ministeranklage.

(9) Dysfunktional ist eine Volksgesetzgebung, die entweder auf die Verfassungsinitiative beschränkt ist (Bundesebene in der Schweiz) oder deren Einleitung nicht hinreichend von der Gesetzesinitiative abhebt (Kalifornien).

(10) Die Ergänzung von Art. 79 Abs. 2 GG um ein obligatorisches Verfassungsreferendum ist empfehlenswert. 